Supporting Information

\title{
Interfacial Modification and Defect Passivation by the Cross-Linking Interlayer for Efficient and Stable CuSCN-Based Perovskite Solar Cells
}

Jinhyun Kim,$^{\dagger}$ Younghyun Lee,$^{\dagger}$ Alan Jiwan Yun, ${ }^{\dagger}$ Bumjin Gil, and Byungwoo Park*

Department of Materials Science and Engineering, Research Institute of Advanced Materials, Seoul National University, Seoul 08826, Korea

\footnotetext{
*E-mail: byungwoo@snu.ac.kr; Phone: +82-2-880-8319; Fax: +82-2-885-9671.

$\dagger$ These authors contributed equally to this work.
} 
Table S1. Carrier lifetimes of perovskites with different HTLs. The lifetimes of free charge carriers are obtained from the time-resolved photoluminescence spectra in Figure $2 b$.

\begin{tabular}{|c|c|c|c|}
\hline HTL & $\tau_{1}[\mathrm{~ns}]^{\mathrm{a}}$ & $\tau_{2}[\mathrm{~ns}]^{\mathrm{a}}$ & $\tau_{\text {avg }}[\mathrm{ns}]^{\mathrm{b}}$ \\
\hline $\mathbf{M A P b I}_{3}$ & 33.6 & 189.2 & 110.1 \\
\hline $\mathrm{MAPbI}_{3} / \mathrm{CuSCN}$ & 2.5 & 12.5 & 4.4 \\
\hline MAPbI/3/PDMS/CuSCN & 1.8 & 8.4 & 2.2 \\
\hline
\end{tabular}


Table S2. Trap density $N_{t}$ and defect energy level $E_{0}$ of CuSCN-based solar cells. Energy levels and densities of the electronic traps obtained from the impedance analysis.

\begin{tabular}{ccc}
\hline HTL & Trap Density $\boldsymbol{N}_{\boldsymbol{t}}\left[\mathrm{cm}^{-3}\right]$ & $\boldsymbol{E}_{\mathbf{0}}[\mathrm{eV}]$ \\
\hline \hline CuSCN & $5.86 \pm 0.57 \times 10^{17}$ & 0.70 \\
\hline PDMS/CuSCN & $2.73 \pm 0.08 \times 10^{17}$ & 0.59 \\
\hline
\end{tabular}


(a) CuSCN wetting time
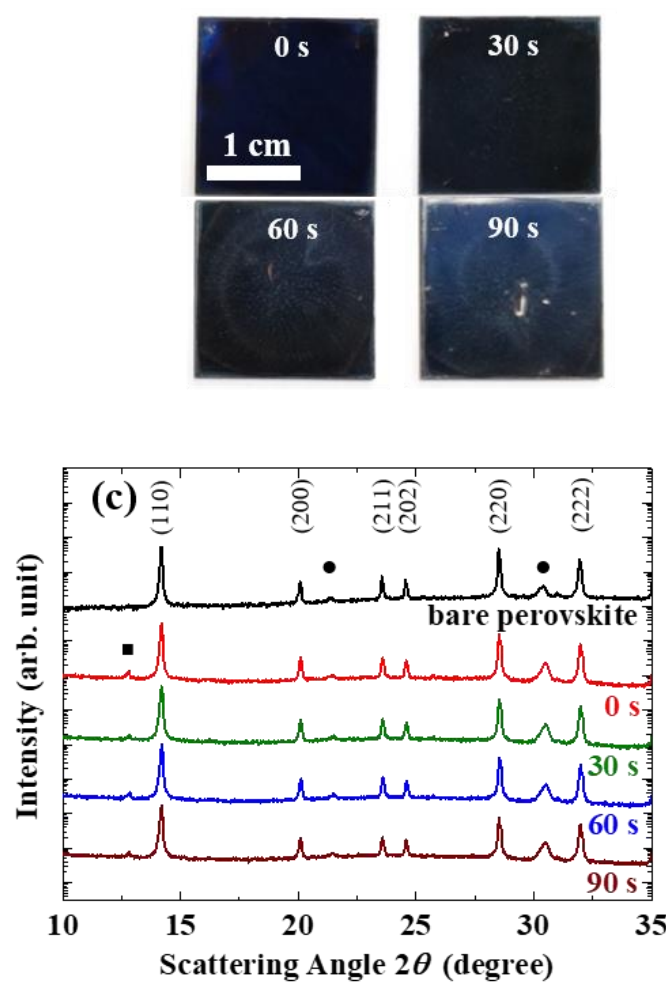

(b)
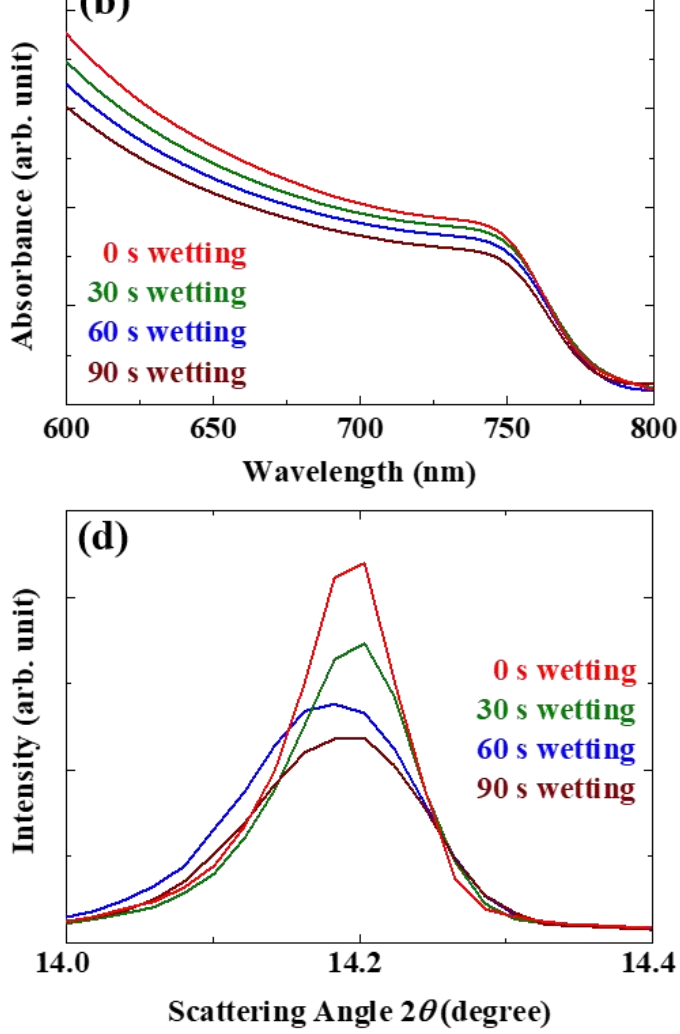

Figure S1. Observation of the perovskite degradation by CuSCN-diethyl sulfide (DES)

solution. (a) Optical images, (b) absorbance spectra, and (c) XRD patterns of $\mathrm{ITO} / \mathrm{SnO}_{2} / \mathrm{MAPbI}_{3} / \mathrm{CuSCN}$ films with different wetting times (from 0 to $90 \mathrm{~s}$ ) of $\mathrm{CuSCN}-\mathrm{DES}$ solution. The square and circles in (c) represent the peaks of $\mathrm{PbI}_{2}$ and ITO, respectively. (d) Comparison of the magnified peaks at $14.2^{\circ}$ (for the (110) plane in $\mathrm{MAPbI}_{3}$ ). 


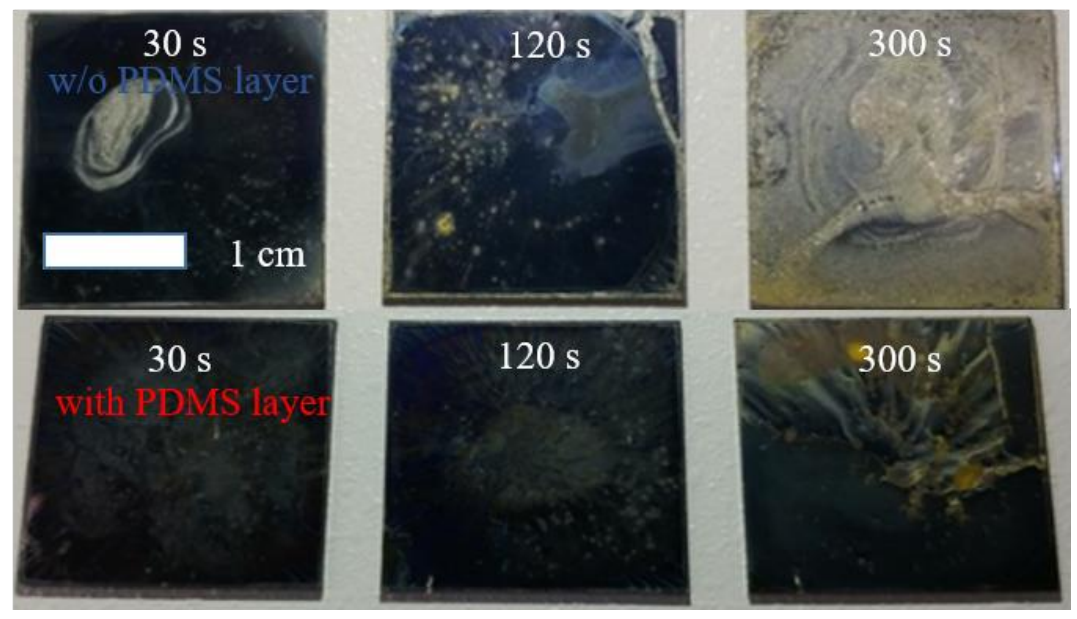

Figure S2. Compared degradation of perovskite by CuSCN-DES solution. Optical images of $\mathrm{ITO} / \mathrm{SnO}_{2} / \mathrm{MAPbI}_{3} / \mathrm{PDMS} / \mathrm{CuSCN}$ films with different wetting times of CuSCN-DES solution, without and with PDMS buffer layer. 

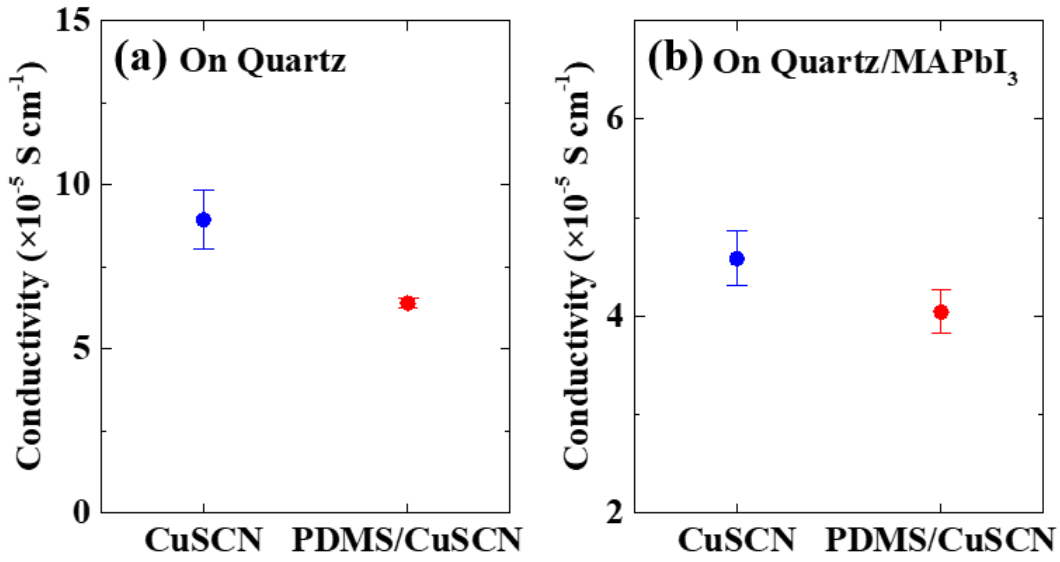

Figure S3. Electrical properties of $\mathrm{CuSCN}$ and PDMS/CuSCN films. Sheet resistance is measured by four-point probe for $\mathrm{CuSCN}$ and PDMS/CuSCN films, which are deposited on (a) quartz substrates and (b) $\mathrm{MAPbI}_{3}$ films. 

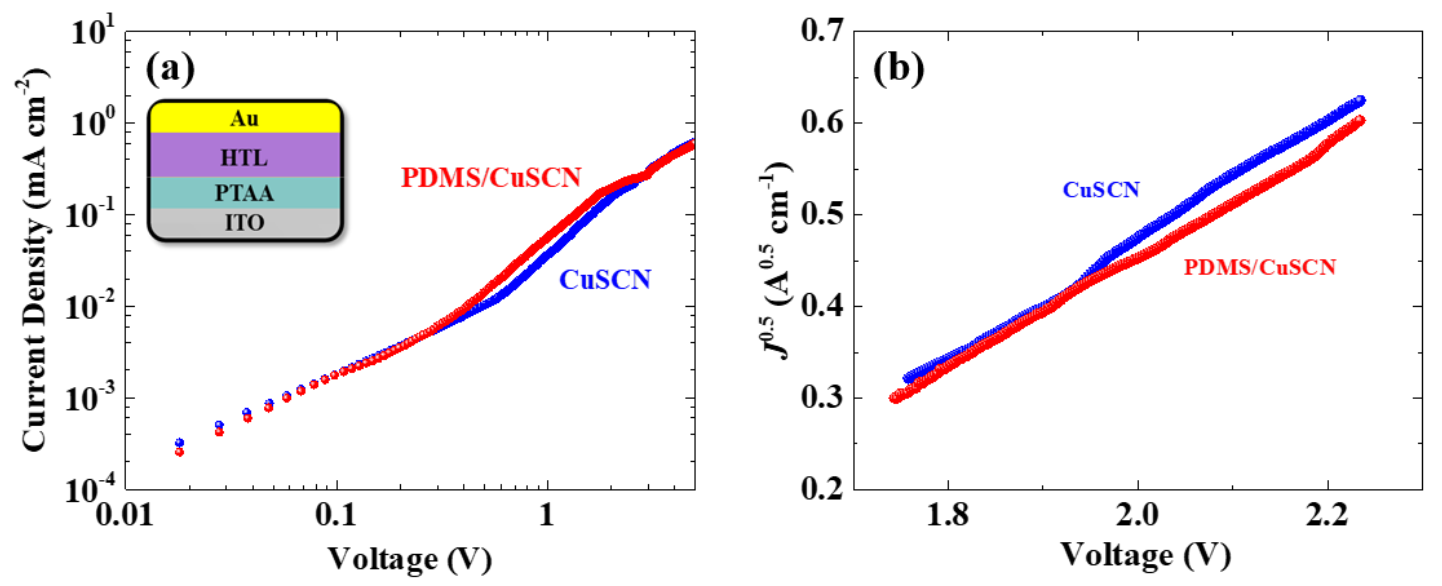

Figure S4. Space-charge limited current (SCLC) measured for CuSCN and PDMS/CuSCN films. (a) $J-V$ characteristics of hole-only devices without perovskite layer, measured in dark condition. (b) $J^{0.5}-V$ plot in the SCLC region with high applied voltage. The hole mobilities $\mu_{h}$ are calculated to be 0.20 and $0.18 \mathrm{~cm}^{2} \mathrm{~V}^{-1} \mathrm{~s}^{-1}$, for CuSCN and PDMS/CuSCN films respectively. 

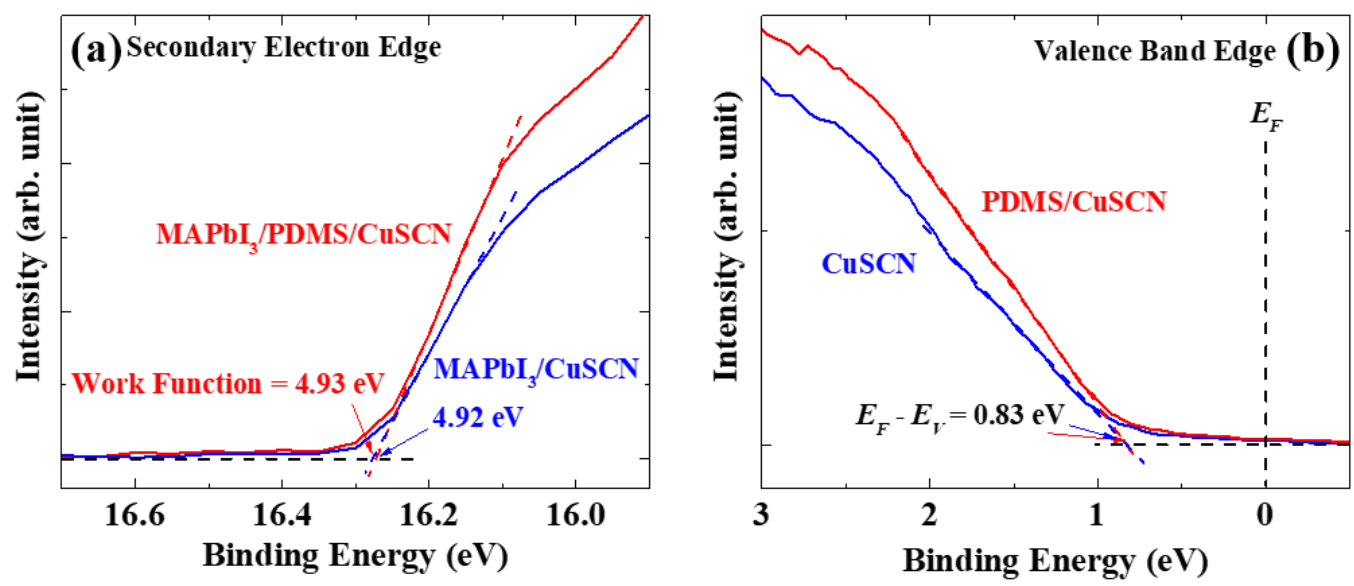

Figure S5. Ultraviolet photoemission spectroscopy measurement for various surfaces.

Photoemission spectra for (a) secondary electron edge and (b) valence band edge on the surfaces of CuSCN HTLs with and without PDMS interlayer. 

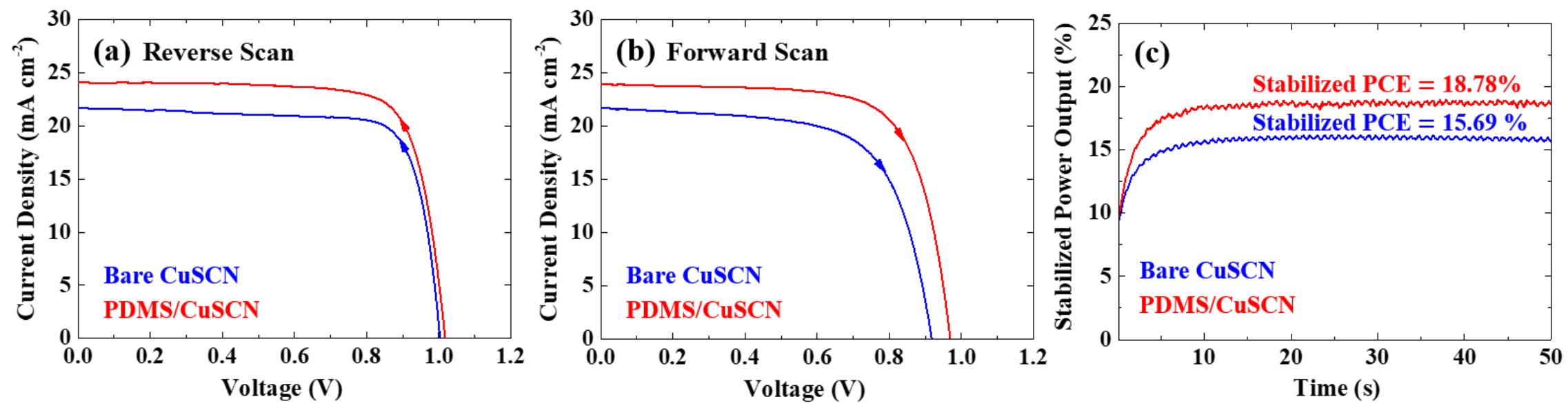

Figure S6. Improved performance of MAPbI 3 -based solar cells by PDMS. $J$ - $V$ curves of MAPbI ${ }_{3}$-based solar cells with (a) reverse and (b) forward scans, and (c) their stabilized power outputs measured at the maximum power voltages. 

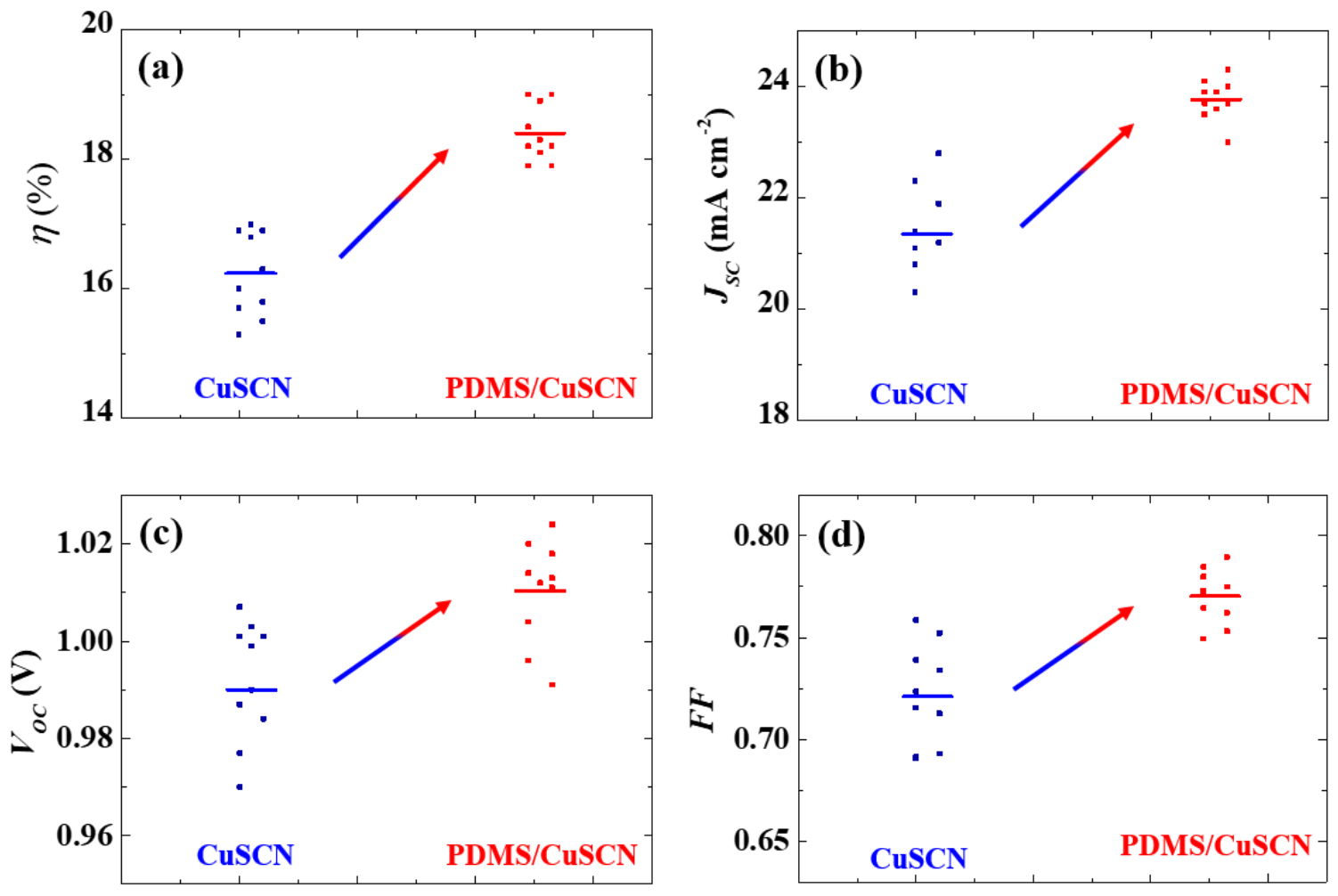

Figure S7. Performance of MAPbI3-based solar cells, without and with PDMS. Comparison of the cell parameters including (a) $\eta$, (b) $J_{S C}$, (c) $V_{O C}$, and (d) $F F$ for $\sim 10$ devices each. 

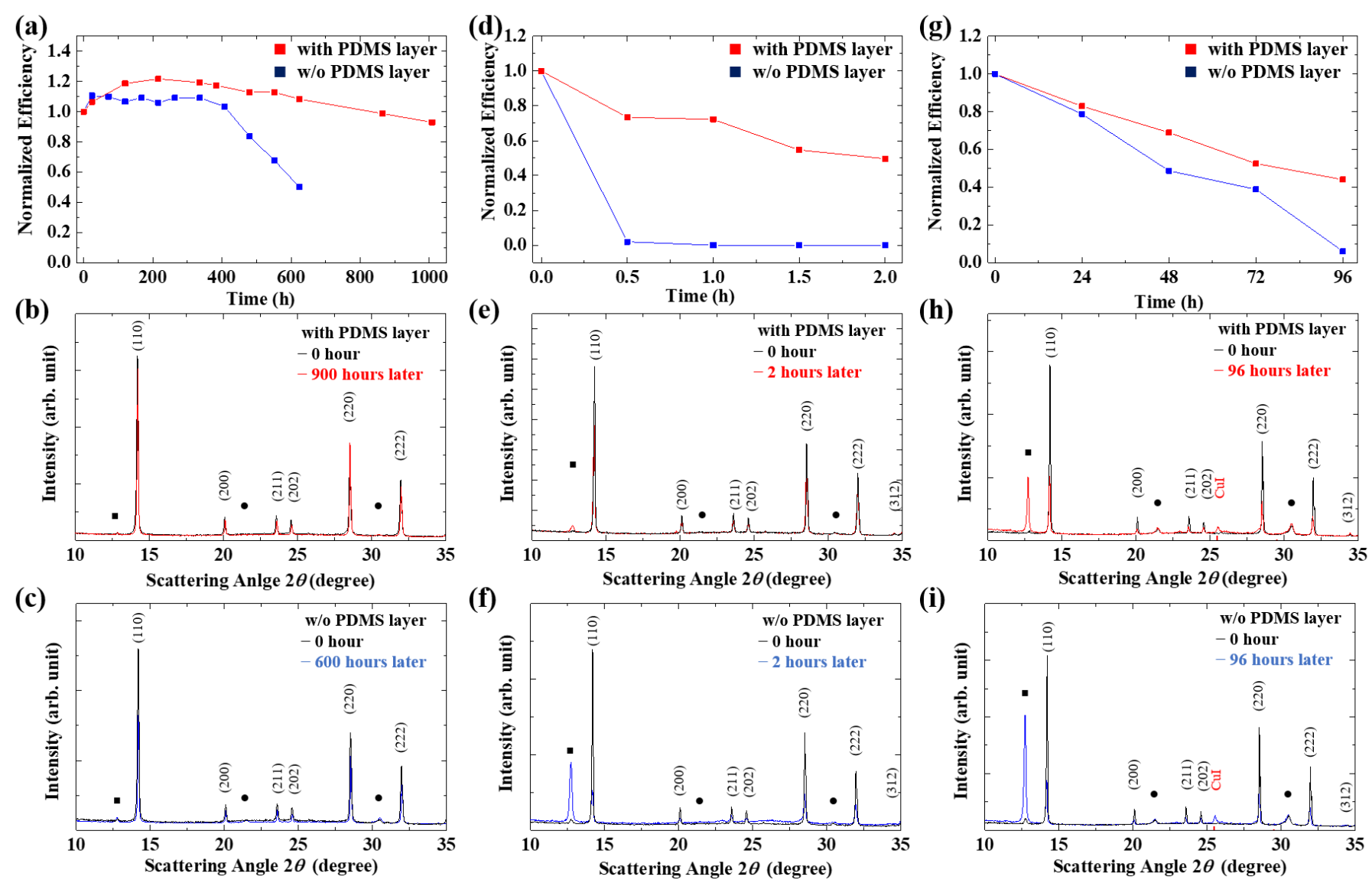

Figure S8. Stabilities of MAPbI3-based solar cells and their XRD patterns before/after the stability tests. (a) Stabilities tested under $25^{\circ} \mathrm{C} / 55 \%$

RH condition and (b,c) XRD patterns of solar cells with and without PDMS interlayer. (d-f) Data analyzed for the solar cells stored under $25^{\circ} \mathrm{C} / 95 \%$

RH conditions and (g-i) under $85^{\circ} \mathrm{C} / 35 \%$ RH conditions. All the tests were conducted for solar cells without encapsulation. 

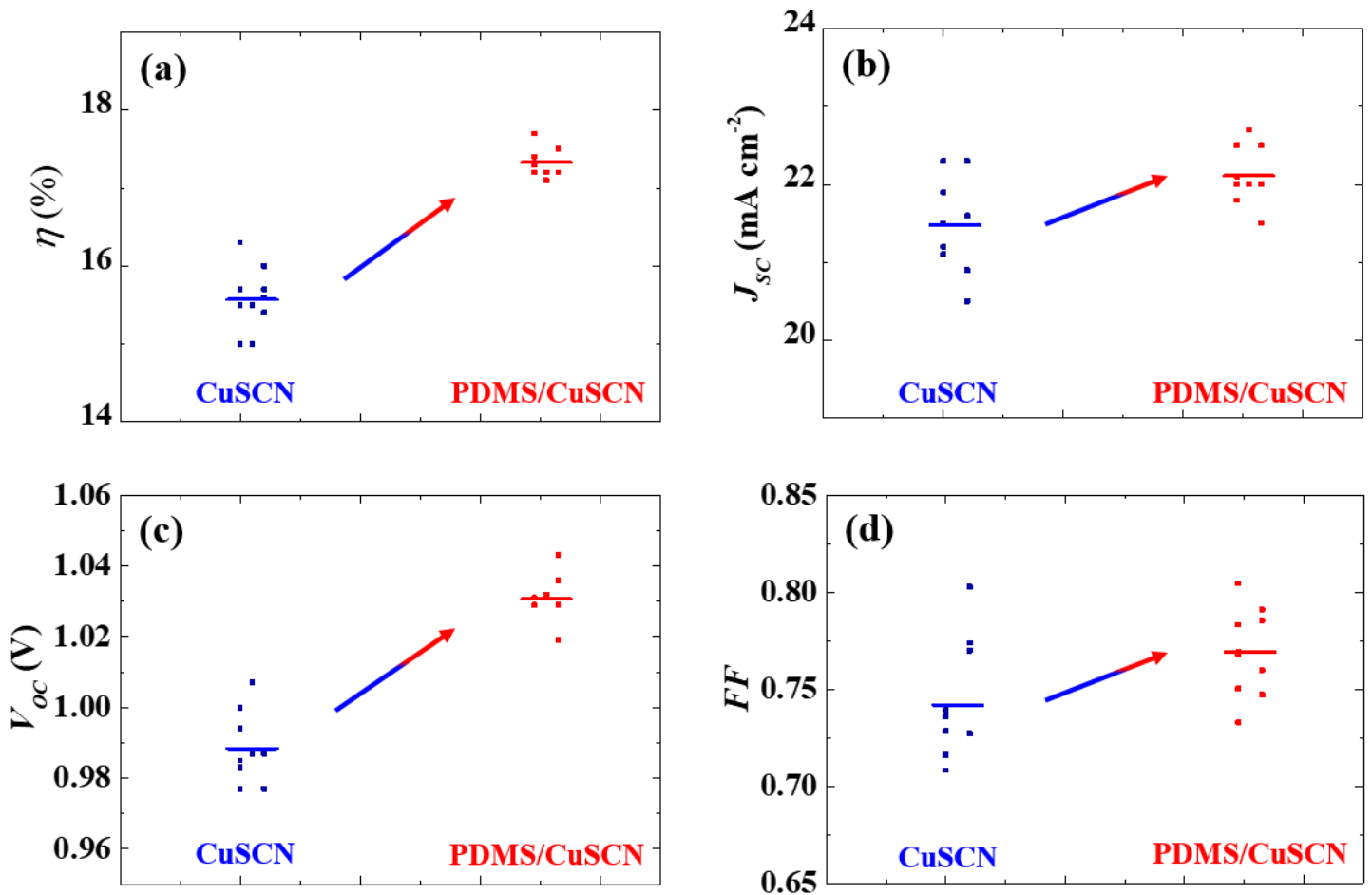

Figure S9. Performance of Cs0.05(FA0.83MA0.17)0.95Pb(I0.83Bro.17)3/CuSCN-based devices. (a) $\eta$, (b) $J_{S C}$, (c) $V_{O C}$, and (d) $F F$ of CsFAMA/CuSCNbased solar cells without and with PDMS interlayer. 

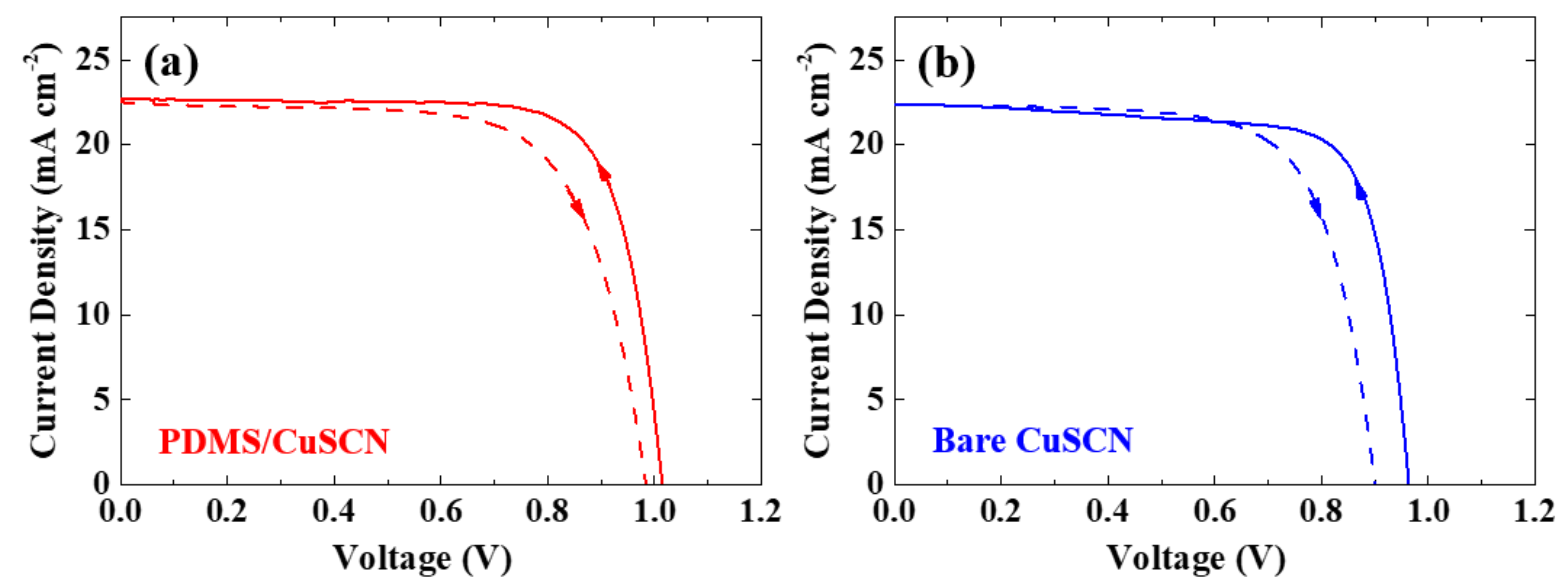

Figure S10. Device performance of CsFAMA-based solar cells. $J$ - $V$ curves of triple-cation perovskite $\left(\mathrm{Cs}_{0.05}\left(\mathrm{FA}_{0.83} \mathrm{MA}_{0.17}\right)_{0.95} \mathrm{~Pb}_{(}\left(\mathrm{I}_{0.83} \mathrm{Br}_{0.17}\right)_{3}\right.$ : CsFAMA)-based solar cells (a) with and (b) without PDMS interlayer. 